\title{
Beam propagation in a Randomly Inhomogeneous Medium
}

\author{
A. A. Stanislavsky* \\ Institute of Radio Astronomy, Ukrainian National Academy of Sciences, \\ 61002 Kharkov, Ukraine \\ Published in Journal of Experimental and Theoretical Physics 98(4), 2004, pp. 705-706
}

\begin{abstract}
Аннотация
An integro-differential equation describing the angular distribution of beams is analyzed for a medium with random inhomogeneities. Beams are trapped because inhomogeneities give rise to wave localization at random locations and random times. The expressions obtained for the mean square deviation from the initial direction of beam propagation generalize the " $3 / 2$ law".
\end{abstract}

PACS: 42.25.Dd, 05.40.-a

Fluctuations of the beam propagation direction in a randomly inhomogeneous medium are frequently observed in nature. Examples include random refraction of radio waves in the ionosphere and solar corona, stellar scintillation due to atmospheric inhomogeneities, and other phenomena. The propagation of a beam (of light, radio waves, or sound) in such media can be described as a normal diffusion process [1, 2]. One extraordinary property predicted for random media - and later revealed - is the Anderson localization [3]. It brings normal diffusion to a complete halt. In the context of wave propagation in a random medium, the Anderson localization is caused by interference of waves resulting from multiple scattering [4]. When two waves propagating in opposite directions along a closed path are in phase, the resultant wave is more likely to return to the starting point than propagate in other directions. The properties of a randomly inhomogeneous medium vary not only from point to point, but also with time. Consequently, localization may take place both at random locations and at random times. Random localization affects diffusive light propagation in a random medium. An approach to description of this effect is developed in this paper.

Suppose that the medium is statistically homogeneous and isotropic. Then, a beam propagating through the medium is deflected at random. Localization implies that the beam is trapped in some region. Since the trapped beam returns to the point where it was trapped, its propagation is "frozen" for some time. After that, a randomly deflected beam leaves the legion and propagates further until it is trapped in another region (or at a point) and the localization cycle repeats. The randomly winding beam path due to inhomogeneities is responsible for the random refraction analyzed in this study.

\footnotetext{
*alexstan@ri.kharkov.ua
} 
The angle $\theta$ of deviation of a beam from its initial direction is characterized by a probability density $W_{\alpha}(\theta, \sigma)$, where $\sigma$ is the path travelled by the beam. Let us derive an integro-differential equation for the probability density. In contrast to rotational Brownian motion, the random walks analyzed here consist of random angle jumps $\Delta \theta_{i}$ at points separated by segments of random length $\Delta \sigma_{i}$. Exact knowledge of the distributions of these random variables is not required. It is sufficient to assume that the angle jumps are independent random variables belonging the domain of attraction of Gaussian probability distribution. The random segment lengths $\Delta \sigma_{i}$ are also identically distributed independent random variables, with stable distribution is characterized by an index $\alpha$. Since $\Delta \sigma_{i}$ is a nonnegative quantity, this distribution is totally asymmetric, and $0<\alpha \leq 1$. Recall that a random variable characterized by a probability distribution $f(x)$, of this kind is described by the Laplace transform

$$
\phi(s)=\int_{0}^{\infty} \exp \{-s x\} d f(x)=\exp \left\{-(A x)^{\alpha}\right\},
$$

where $x \geq 0$ and $A>0$ [5]. The total path length is the sum of all $\Delta \sigma_{i}$. Both $\Delta \sigma_{i}$ and $\Delta \theta_{i}$ are Markov processes. However, since the former is the master process with respect to the latter, the resultant process may not preserve the Markov property [6]. On account of convergence of distributions we can definitely pass from the discrete model to a continuous limit [7]. This leads to the diffusion equation

$$
W_{\alpha}(\theta, \sigma)-W_{\alpha}(\theta, 0)=\int_{0}^{\sigma} \frac{D}{\Gamma(\alpha) \sin \theta} \frac{\partial}{\partial \theta}\left(\sin \theta \frac{\partial W_{\alpha}\left(\theta, \sigma^{\prime}\right)}{\partial \theta}\right)\left(\sigma-\sigma^{\prime}\right)^{\alpha-1} d \sigma^{\prime},
$$

where $D$ is a diffusion coefficient and $\Gamma(x)$ is the gamma function. The solution to this equation can be expressed as an integral transform of the probability density associated with rotational Brownian motion:

$$
W_{\alpha}(\theta, \sigma)=\int_{0}^{\infty} F_{\alpha}(z) W_{1}\left(\theta, \sigma^{\alpha} z\right) d z
$$

where

$$
F_{\alpha}(z)=\sum_{k=0}^{\infty} \frac{(-z)^{k}}{k ! \Gamma(1-\alpha-k \alpha)} .
$$

The diffusion equation yields the mean

$$
\overline{\cos \theta}=E_{\alpha}\left(-2 D \sigma^{\alpha}\right),
$$

where

$$
E_{\alpha}(-x)=\sum_{n=0}^{\infty}(-x)^{n} / \Gamma(1+n \alpha)
$$

is the Mittag-Leffler function. At large $\sigma$, all beam directions are equiprobable. However, in contrast to normal diffusion $(\alpha=1)$, a beam has to travel a longer path $\sigma$ to reach this state. This process is somewhat analogous to "superslow" relaxation.

Following the method developed in [8], one can find the mean square of the distance $r$ from the starting point to the observation point reached by the beam that has travelled an intricate path of length $\sigma$ through the medium:

$$
\overline{r^{2}}=\frac{\sigma^{\alpha}}{D \Gamma(\alpha+1)}-\frac{1}{2 D^{2}}\left(1-E_{\alpha}\left(-2 D \sigma^{\alpha}\right)\right) .
$$


If $D \sigma \ll 1$, then

$$
\overline{r^{2}} \approx 2 \sigma^{2 \alpha}\left(\frac{1}{\Gamma(2 \alpha+1)}-\frac{2 D \sigma^{\alpha}}{\Gamma(3 \alpha+1)}\right) .
$$

If the $z$ axis of a polar coordinate system is aligned with the initial beam direction, then the mean square of the distance passed by the beam along this axis is given by the formula

$$
\overline{z^{2}}=\frac{1}{3 D}\left[\frac{\sigma^{\alpha}}{\Gamma(\alpha+1)}-\frac{1}{6 D}\left(1-E_{\alpha}\left(-6 D \sigma^{\alpha}\right)\right)\right] .
$$

If $D \sigma$ is small, then

$$
\overline{z^{2}} \approx 2 \sigma^{2 \alpha}\left[\frac{1}{\Gamma(2 \alpha+1)}-\frac{6 D \sigma^{\alpha}}{\Gamma(3 \alpha+1)}\right] .
$$

Now, the mean square deviation of the beam from its initial direction can be calculated by combining (11) with (3):

$$
\overline{\rho^{2}}=\overline{r^{2}}-\overline{z^{2}}=\frac{2 \sigma^{\alpha}}{3 D \Gamma(\alpha+1)}-\frac{1}{2 D^{2}}\left(1-E_{\alpha}\left(-2 D \sigma^{\alpha}\right)\right)+\frac{1}{18 D^{2}}\left(1-E_{\alpha}\left(-6 D \sigma^{\alpha}\right)\right) .
$$

If $D \sigma$ is small, then a generalized " $3 / 2$ law" is obtained [8]:

$$
\sqrt{\overline{\rho^{2}}} \approx \frac{2 \sqrt{2}}{\sqrt{\Gamma(3 \alpha+1)}} D^{1 / 2} \sigma^{3 \alpha / 2} .
$$

The mean squares given by (11), (3) and (15) increase as $\sigma^{\alpha}$ at large $\sigma$. The case of $\alpha=1$ corresponds to normal diffusion without wave localization. Thus, the approach developed here embraces classical results of the theory of beam propagation medium in a randomly inhomogeneous medium [1, 2].

Finally, it should be recalled that experimental deviations from the " $3 / 2$ law" (more precisely, from an exponent of $3 / 2$ in the classical power law) were mentioned in [9]. However, they were attributed to systematic measurement errors, probably because of the lack of plausible interpretation. This problem can be revisited in view of the results

obtained in this study. Moreover, new accurate experimental studies of beam propagation in appropriate randomly inhomogeneous media would be extremely useful for verifying the model proposed here.

\section{Список литературы}

[1] S. M. Rytov, Introduction to Statistical Radiophysics (Nauka, Moscow, 1966).

[2] L. A. Chernov, Wave Propagation in a Random Medium (Nauka, Moscow, 1975; McGraw-Hill, New York, 1960).

[3] P. W. Anderson, Phys. Rev. 109, 1492 (1958).

[4] D. S. Wiersma, P. Bartolini, A. Lagendijk, R. Righini, Nature 390, 671 (1997).

[5] V. M. Zolotarev, One-Dimensional Stable Distributions (Nauka, Moscow, 1983; Am. Math. Soc., Providence, RI, 1986). 
[6] W. Feller, An Introduction to Probability Theory and Its Applications, 3rd ed. (Wiley, New York, 1967; Mir, Moscow, 1964).

[7] A. A. Stanislavsky, Phys. Scripta 67, 265 (2003).

[8] L. A. Chernov, Zh. Eksp. Teor. Fiz. 24, 210 (1953).

[9] I. G. Kolchinskii, Astron. Zh. 29, 350 (1952). 